\author{
L.A. Nesterenkova, P.A. Nesterenkov, Z.H. Spabekova \\ Al-Farabi Kazakh National University, Almaty, Kazakhstan, \\ e-mail: stolkner@gmail.com

\section{ON TWO WAYS TO IMPROVE THE RHEOLOGY OF HIGH VISCOUS OIL IN AN OIL PIPELINE}

\begin{abstract}
Transportation of highly viscous and high-curing oils through main pipelines requires significant energy costs. Thus, the task of choosing the cheapest pumping modes is very relevant. The article describes and proposes a solution to the oil flow problem in a pipeline using two methods: with preheating and using a Laval nozzle at the inlet of the pipeline. Mathematical models of the flow of highviscosity oil in the main oil pipeline for the two named pumping methods have been compiled. An algorithm has been developed for calculating temperature, viscosity and pressure along the length of the Uzen-Atyrau pipeline at various oil flow rates. The results of temperature and pressure distribution are analyzed and compared at different oil flow rates along the length of the pipeline for two pumping methods. It is shown that the use of cavitation improves the rheological properties of oil and can significantly reduce the cost of pumping. The research results can be used to predict the operation of main oil pipelines pumping oil both in a heated state and in isothermal mode with a Laval nozzle.
\end{abstract}

Key words: high viscosity oil, pressure and temperature along the pipeline, cavitation, oil pipelines operation costs.

\section{Introduction}

Kazakhstan has the largest deposits of liquid hydrocarbons. In terms of oil reserves, our country is one of the 15 leading countries in the world, and has $3.3 \%$ of the world's carbon reserves [1].

The bulk of the oil produced in our country is highly viscous, because it contains a large number of paraffin fractions, resins, asphaltenes and other components. Pipeline transport of such oils require significant energy costs [2].To reduce them, special methods are used to improve the rheological properties of the transported product [2,3]. For example, in the practice of transporting highly viscous high-hardening oils and petroleum products, methods such as pumping in a carrier stream have found application (in this method, oil is cooled to a level where it will be a solid granular body and transferred in a stream of liquefied gas); pipeline transport with continuous heating (using various coolants or electric heating of the pipeline); pumping with diluents (with dilution of oil with external low-viscosity oils, liquefied gas, etc.); hydro-transport (pumping oil with a near-wall water layer); pumping heat-treated oils with improving their rheological properties; preheating pumping and so on [3].

(C) 2020 al-Farabi Kazakh National University

\section{Material and methods}

Among the existing methods, the pre-heated pumping method has gained the greatest popularity, which can significantly reduce the viscosity of the transported product $[3,4]$. In this method of transportation, oil (usually $45-65^{\circ} \mathrm{C}$ ) heated to the optimum temperature is pumped into the main line by pumps. Through certain distances, intermediate heat and pumping stations are established along the pipeline route, where the oil that cools down along the way is heated and pumped to the next station. The distance between pumping stations and thermal stations is determined by the rate of temperature decrease and pressure depression.

The operation of underground heated oil pipelines has a number of related features. first of all, with the influence of the conditions of their heat exchange with the environment. In many cases, there are reasons that adversely affect the temperature regime of the pipeline (annual changes in soil temperature; shutdowns and shutdowns of pumping and heating units, both planned and due to equipment failures, triggering of the automatic protection system; change in the thermal conductivity of the soil due to strong rains, intense melting of snow, floods, etc.). This creates a danger Int. j. math. phys. (Online) 
of emergency situations and requires additional capital investments.

Due to the fact that pipelines are being built in regions with different climatic and environmental conditions, problems arise in the selection and justification of laying methods and operation modes of pipelines. When designing them, the selection of the main technological and structural parameters and construction solutions is carried out mainly on the basis of the conditions of stationary operation of the pipeline. This necessitates further improvement of the methods for studying the transport of oil and oil products. In this regard, the issue of using nonstandard methods for changing the rheological properties of oils and petroleum products becomes relevant.

One of promising methods for changing the viscosity of oil is cavitation $[5,6]$. For cavitation to occur, a hollow cylindrical pipe of variable section (tapering, and then expanding part in a certain way) is placed in the pipeline. The fact is that when oil passes through this pipe, called the Laval nozzle, due to cavitation, as a result of a decrease in pressure in the expanding part of the nozzle, a sharp change in its rheological properties occurs. At the outlet of the Laval nozzle, oil has a lower viscosity, higher fluidity, and lower tensile stress [7].

This paper explores the possibility of using a Laval nozzle at the inlet of the pipeline to create cavitation effects of the transported liquid, which can reduce the viscosity of oil by comparing the calculations of the flow of oil through a pre-heated oil pipeline and through a pipeline with a Laval nozzle.

\section{Results and discussion}

In a preheated oil pipeline, a steady nonisothermal oil flow in linear sections between intermediate stations is described by a system of differential equations of motion, continuity and energy $[3,4]$.

When constructing a mathematical model of the steady non-isothermal flow of highly viscous fluid in an underground pipeline laid over rough terrain, the following assumptions were made:

- due to the large length of the main pipelines, we use a one-dimensional mathematical model of the flow, the $\mathrm{X}$ axis is compatible with the axis of the pipe, and all process parameters are averaged over the pipe section;
- thermal and pumping stations located along the route are considered point-like because of their small size compared to the length of the pipeline;

- the pipeline is divided into sections between thermal and pumping stations (so called "linear sections" in this work);

- along the length of the pipeline there are no intermediate sources of mass;

- oil is considered as a single-phase viscoplastic incompressible fluid;

- heat transfer between the pipeline and the surrounding soil obeys the Newton-Richmann law;

- the coefficients of density $\rho$, specific heat of oil $c$ and soil thermal conductivity $\lambda$ are independent of temperature.

Under the assumptions made, the steady nonisothermal flow of oil in the linear sections between intermediate stations will have $[3,4]$ :

$$
\left.\begin{array}{c}
\frac{d p}{d x}=-\rho \cdot g \cdot\left(\frac{d z}{d x}+\frac{d h_{\mathrm{Tp}}}{d x}\right) \\
\frac{d w}{d x}=0 ;
\end{array}\right\}
$$

with boundary conditions

$$
\mathrm{p}(0)=\mathrm{p}_{\mathrm{n}} ; \mathrm{T}(0)=\mathrm{T}_{\mathrm{n}} ; \mathrm{w}(0)=\mathrm{w}_{\mathrm{n}}
$$

and interfacing conditions at intermediate stations:

$$
p_{j}^{+}=p_{j}^{-}+\Delta p_{j} ; T_{j}^{+}=T_{j}^{-}+\Delta T_{j}
$$

where $\mathrm{p}$ - pressure in the pipeline, $\mathrm{w} ; \mathrm{T}$ - respectively, the flow velocity and oil temperature; D - internal diameter of the pipeline, $\mathrm{z}-$ geodetic height of the pipeline; $\mathrm{T}_{\text {окр }}$ - temperature of the soil surrounding the pipeline; $k=2 \lambda /(\mathrm{D} \cdot \ln (4 \mathrm{~h} / \mathrm{D}))-$ heat transfer coefficient from the oil pipeline to the surrounding soil; $\mathrm{h}$ - laying depth to the pipe axles, $\Delta \mathrm{T}_{\mathrm{j}}, \Delta \mathrm{p}_{\mathrm{j}}$; changes in temperature and pressure at the $\mathrm{j}^{\text {th }}$ station, the $\mathrm{j}$ index indicates the station number; the indices "+" and "-"indicate the values of the values on the right and left border of the section; $\mathrm{N}$ - number of stations and linear sections between stations.

To determine the frictional pressure loss of viscoplastic oil $\mathrm{h}_{\mathrm{тр}}$, will use the Darcy - Leibenzon expression $[3,8]$ : 


$$
\begin{aligned}
\frac{d h_{\mathrm{T} p}}{d x} & =\rho \cdot g \cdot \beta \cdot\left(\frac{\pi \cdot w}{4}\right)^{2-m} . \\
& \cdot \frac{v^{m}}{D^{m+1}}+\frac{16}{3} \cdot \frac{\tau_{0}}{\rho \cdot g \cdot D}
\end{aligned}
$$

where $m$ and $\beta$ are coefficients depending on the flow regime [3].

The kinematic viscosity coefficient $v$ and the ultimate shear stress $\tau_{0}$ are largely dependent on temperature. For an analytical description of these dependencies, we use the expressions [3]:

$$
\begin{gathered}
v=v\left(T_{1}\right) \cdot \exp \left[-a_{1} \cdot\left(T-T_{1}\right)\right] ; \\
\tau_{0}=\tau_{0}\left(T_{1}\right) \cdot \exp \left[-a_{2} \cdot\left(T-T_{1}\right)\right]
\end{gathered}
$$

where $\alpha_{1}, \alpha_{2}$ are empirical constants, $T_{1}$ is a fixed temperature.

The cost of operating the pipeline is mainly determined by fuel consumption level at thermal stations and electricity consumption at pumping stations. For a stationary flow regime, these costs are determined by the expression [4]:

$$
\begin{gathered}
S=S_{n}+S_{T}= \\
=w \cdot t \cdot\left(\sum_{j=0}^{N} \varphi_{j} \cdot \Delta p_{j}+\sum_{j=0}^{N} \xi_{j} \cdot \Delta T_{j}\right)
\end{gathered}
$$

where $\mathrm{t}$ - operating time of the pipeline; $\varphi_{\mathrm{j}}, \xi_{\mathrm{j}}$ are, respectively, values proportional to the cost of a unit of fuel and electricity of the $\mathrm{j}^{\text {th }}$ station. If the price of fuel and electricity at all stations is the same, the cost functional (7) taking into account (4) will be minimal under the following conditions [4]:

$$
T_{j}^{-}=T_{\text {min }} ; p_{j}^{-}=p_{\text {min }} ; j=\overline{1, N+1}
$$

where $\mathrm{T}_{\min }, \mathrm{p}_{\min }-$ the minimum allowable temperature and pressure in the pipe.

From the continuity equation from system (1) we have: $w(x)=$ const $=w_{n}$.

The energy equation (2) on the linear sections of the pipeline has an analytical solution:

$$
T(x)=T_{\text {окр }}+\left(T_{j}^{+}-T_{\text {окр }}\right) \cdot \exp \left(-\frac{4 \cdot k \cdot x}{\rho \cdot c \cdot D \cdot w}\right)
$$

The equation of motion from system (1), taking into account (5), (6), contains large nonlinearities, therefore, the pressure distribution along the pipeline route will be calculated numerically using finite-difference schemes. Having divided each linear section $j$ into segments of length $\Delta x_{j}$, and taking into account the boundary conditions, we find the temperature from (9) at each node and then the pressure from (1), (3), (4):

$$
\begin{gathered}
T_{J}^{I}=T_{\text {окр }}+\left(T_{j}^{I-1}-T_{\text {окр }}\right) \cdot \exp \left(-\frac{4 \cdot k \cdot \Delta x_{j}}{\rho \cdot c \cdot D \cdot w}\right) \\
p_{J}^{I}=p_{J}^{I-1}-\rho \cdot g \cdot\left(z_{J}^{I}-z_{J}^{I-1}\right)+\left\{\rho \cdot g \cdot \beta \cdot\left(\frac{\pi \cdot w}{4}\right)^{2-m} \cdot\right. \\
\left.+\frac{\left.v\left(T_{1}\right) \cdot \exp \left[-a_{1} \cdot\left(T_{\text {окр }}+\left(\frac{T_{j}^{I-1}+T_{j}^{I}}{2}-T_{\text {окр }}\right) \cdot \exp \left(-\frac{4 \cdot k \cdot \Delta x_{j}}{\rho \cdot c \cdot D \cdot w}\right)-T_{1}\right)\right]^{m}\right]}{D^{m+1}}\right]+ \\
\left.+\frac{16}{3 \cdot D} \cdot \tau_{0}\left(T_{1}\right) \cdot \exp \left[-a_{2}\left(T_{\text {окр }}+\left(\frac{T_{j}^{I-1}+T_{j}^{I}}{2}-T_{\text {окр }}\right) \cdot \exp \left(-\frac{4 k \Delta x_{j}}{\rho c D w}\right)-T_{1}\right)\right]\right\} \cdot \Delta x_{j} ; \\
\mathrm{p}_{1}^{0}=p_{n} ; T_{1}^{0}=T_{n} ; w_{1}^{0}=w_{n}=\operatorname{const} ; \\
p_{j}^{0}=p_{j-1}^{M_{j-1}+\Delta p_{j} ; T_{j}^{0}=T_{j-1}^{M{ }_{j-1}}+\Delta T_{j}} ; \\
i=\overline{0, M_{J}}, j=\overline{0, N+1}
\end{gathered}
$$


where $M_{j}$ is the number of partitions on the $j^{\text {th }}$ segment.

The algorithm for solving the thermo hydraulic fuel and energy problem (10) taking into account the cost of costs (7) is implemented as aapplication software. The calculation results for the UzenAtyrau trunk pipeline are shown in Figures 1,2.

In an oil pipeline with a Laval nozzle at the inlet, the mathematical model will be slightly different from model (1) - (10).

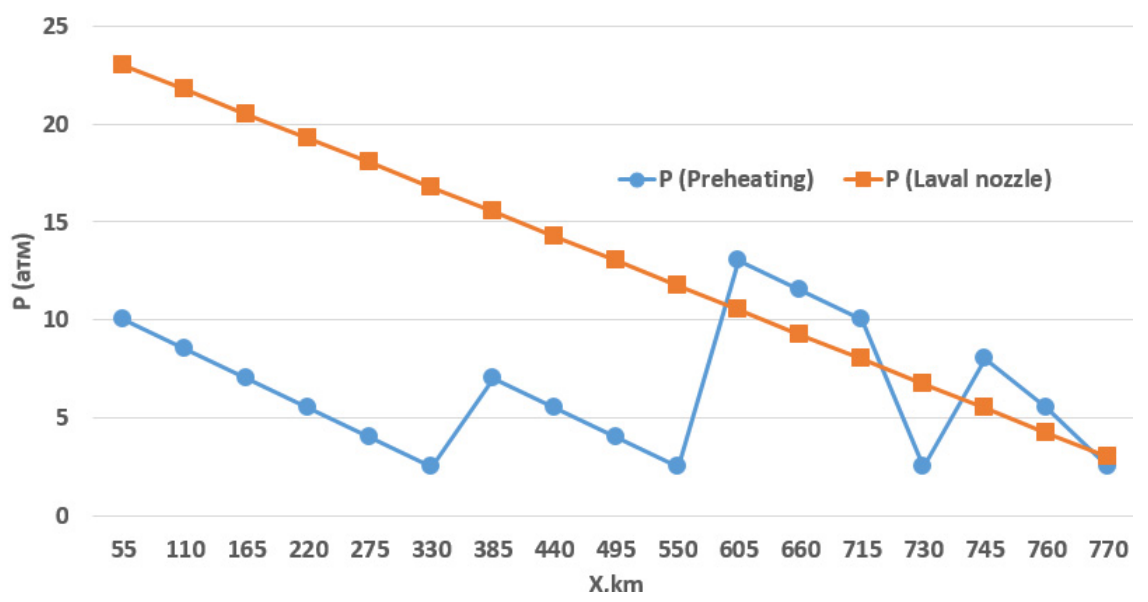

Figure 1 -Pressure distribution along the Uzen-Atyrau oil pipeline

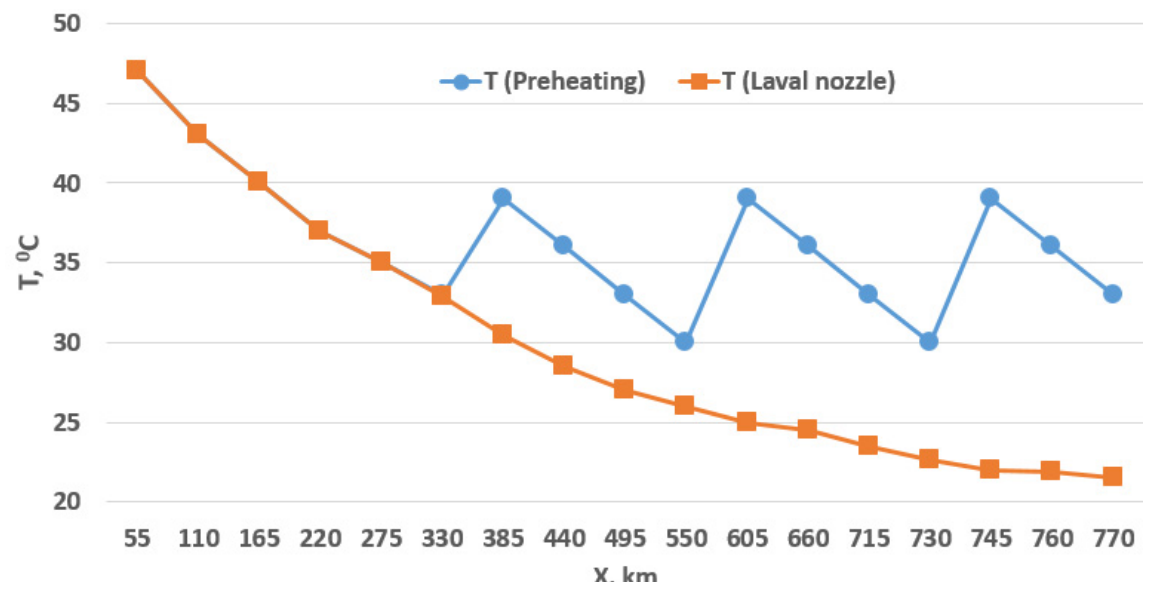

Figure 2 - Temperature distribution along the Uzen-Atyrau oil pipeline

Firstly, oil heating at intermediate stations is not required, because its viscosity decreases as it passes through the Laval nozzle. The decrease in viscosity depends on the nozzle entry angle $\alpha$ and the initial temperature $T_{n}$. The empirical formula has the form:

$$
v=\left(f \cdot a^{2}+y \cdot \alpha+n\right) \cdot \exp \left(u \cdot T_{n}\right)
$$

where $\mathrm{f}, \mathrm{y}, \mathrm{n}, \mathrm{u}$ - are empirical constants.

Note that the viscosity in this case is constant, and oil is a viscoplasticfluid for which $d h_{T p} / d x$ are described by expression (5).
Secondly, the effect of cavitation and a change in the cross section of the pipe leads to:

$$
\tau_{0}=0, \nu=\text { const }
$$

and pressure losses, which can be calculated by the formulas $[6,9,10]$ :

$$
\left.\begin{array}{c}
d p_{1}=\gamma_{1} \cdot \frac{D}{D_{\min }} \cdot \rho \cdot\left(\frac{w_{\mathrm{\kappa p}}-w}{2}\right)^{2} ; \\
d p_{2}=d p_{\text {кав }}=\gamma_{2} \cdot \frac{p-p_{\text {кав }}}{\frac{\rho \cdot w_{\mathrm{Kp}}^{2}}{2}} ;
\end{array}\right\}
$$


where $\gamma_{1}, \gamma_{2}$ are empirical constants; $\mathrm{w}_{\text {кр }}, \mathrm{p}_{\text {кав }}$ are the critical values of pressure and velocity at which cavitation occurs; $D_{\min }$ is the minimum diameter in the Laval nozzle.

Thirdly, as the experiment [7] showed, the decrease in viscosity is so significant, that for sufficiently long pipelines intermediate pumping stations can be abandoned using only the head one.

Thus, the stationary process of oil transportation in this case will be described by the system of equations (1), (2), (5), (11), (12), and the linear section is the entire pipeline and $\mathrm{N}=1$. The boundary conditions taking into account (12) are given in the form:

$$
\left.\begin{array}{c}
p_{1+}(0)=\Delta p_{0}-d p_{1}-d p_{2} \\
T_{1}^{+}(0)=T_{n} ; w(0)=w_{n}
\end{array}\right\}
$$

Since in this case there are no nonlinearities in the model, problem (1), (2), (5), (11) - (13) is solved analytically:

$$
\left.\begin{array}{c}
p(x)=p_{0}^{+}-\frac{\rho \cdot g \cdot \beta}{D^{m+1}}\left(\frac{\pi \cdot w}{4}\right)_{w=\text { const }}^{2-m} \cdot v^{m} \cdot x-\rho \cdot g \cdot\left(z(x)-z_{0}\right) ; \\
T(x)=T+\left(T_{0}^{+}-T\right) \cdot \exp \left(-\frac{4 \cdot k \cdot x}{\rho \cdot c \cdot D \cdot w}\right) .
\end{array}\right\}
$$

The cost of the pipeline operation (7) in this case will be:

$$
S=S_{n}=w \cdot t \cdot\left(\varnothing_{0} \cdot \Delta p_{0}\right)
$$

and the minimum cost will be achieved with $\mathrm{p}_{1}=$ $\mathrm{p}_{\min }$.

The results of calculations of the oil flow in the pipeline using a Laval nozzle are shown in figures 1,2 .

Comparison of the results of calculations of the oil pipeline with preheating and using cavitation at the inlet of the pipeline showed (see Figures 1-3) that its application allows to operate without intermediate heating stations and with much lower energy costs, which is economically very profitable. So, in the case shown in the graphs, the use of a nozzle allows reducing the cost of transporting highviscosity oil by 3 times.

However, it should be noted that when using a Laval nozzle, high-viscosity oil recovers its original structure over time [5], which can somewhat reduce the positive effect of cavitation.

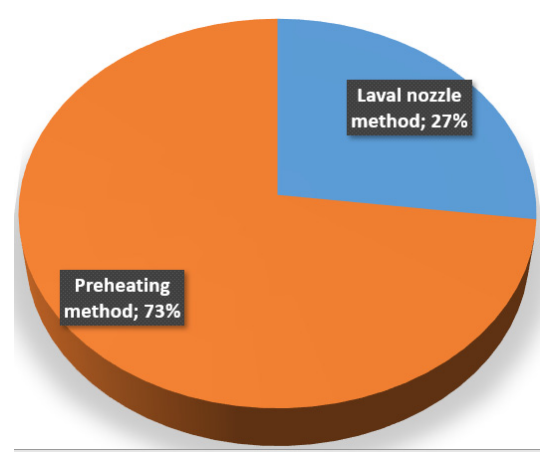

Figure 3-Comparison of operationcost of the Uzen-Atyrau oil pipeline

\section{Conclusions}

Mathematical models of the flow of highviscosity oil in the main oil pipeline for two pumping methods have been compiled: with preheating and using a Laval nozzle at the pipeline inlet.

An algorithm has been compiled to calculate the temperature, viscosity and pressure along the length of the Uzen-Atyrau pipeline at different oil flow rates.

The paper analyzes and compares the results of the distribution of temperature and pressure at different speeds of oil flow along the length of the pipeline for two pumping methods.

It was found that the viscosity of cavitationtreated oil is four times less than the initial value, which can significantly reduce the cost of pumping.

\section{References}

1. Cherdabaev R. Oil of Kazakhstan, Astana: "Aldongar", 2012.

2. Abalkin, "Status of pipeline projects in Kazakhstan for December 2015". polismi.org, published 12/31/2015,

http://forum.polismi.org/index.php?/topic/132 88(accessed 10/17/2019).

3. Agapkin V.M., Krivoshein B.L., Yufin V.A. Thermal and hydraulic calculations of pipelines for oil and oil products, Moscow: Nedra, 1981.

4. Nesterenkova L.A., Nesterenkov P.A. Minimization of the cost of a non-isothermal oil pipeline. VestnikKazNITU, 2 (2019): 528 - 535.

5. Smorodov E.A., Galiakhmetov R.N., Ilgamov M.A. Physics and Chemistry of Cavitation. Moscow: Science, 2008. 
6. "Cavitation drip fluid flow", firing-hydra.ru, https://firing-hydra.ru/index.

php?request $=$ full\&id=406, (accessed 10/17/2019).

7. Nadirov N.K., Kovachuk T.N., Dukhovny T.S., et al. Patent 788 Republic of Kazakhstan MKI E!17O 1/16 "Method for the preparation of highviscosity oils for pipeline transportation" (№ 231939/1, published 04.15.1994).

8. Nesterenkova L. A. Mathematical modeling of non-isothermal flow of high-paraffin oil in the pipeline.Higher School of Kazakhstan, Information Technologies 2 (2013): 156-159.
9. KamenevaT.Yu., Nesterenkova L.A. A numerical study of the reduction of oil viscosity under the influence of cavitation, Abstracts of the Kazakhstan-Russian scientific-practical conference "Mathematical modeling - scientific, technological and environmental problems in the oil and gas industry", Almaty, 1997, p. 96.

10. Hafizov I.F., Aleksandrov A.A., Suschev S.P., et al. Modeling and calculation method of cavitation-vortex apparatus, Vestnik MGTU named after N.E. Bauman (Natural Sciences) 1 (2017): 7891. 